\title{
CHUVA DE SEMENTES EM FRAGMENTO DE FLORESTA ATLÂNTICA SEMIDECÍDUA
}

\author{
Cristiane Reis Barcelos SILVA ${ }^{1}$ \\ Fábio Francisco da SILVA ${ }^{2}$ \\ Eric Batista FERREIRA ${ }^{3}$ \\ Marcelo POLO ${ }^{4}$
}

\begin{abstract}
${ }^{1}$ Licenciada em Ciências biológicas, Universidade Federal de Alfenas (Unifal-MG). E-mail: crisreisb@bol.com.br ${ }^{2}$ Mestre em Ecologia e Tecnologia ambiental, Universidade Federal Alfenas. E-mail: fa_biounifal@yahoo.com.br ${ }^{3}$ Doutor em Estatística, professor adjunto III, Instituto de Ciências Exatas, Universidade Federal de Alfenas. E-mail: eric.ferreira@unifal-mg.edu.br.

${ }^{4}$ Doutor em Biologia Vegetal, professor adjunto IV, Instituto de Ciências da Natureza, Universidade Federal Alfenas. E-mail: marcelo.polo@unifal-mg.edu.br
\end{abstract}

Recebido em: 29/05/2014 - Aprovado em: 30/06/2014 - Disponibilizado em: 30/07/2014

Resumo: A sobrevivência e a dinâmica das florestas dependem em grande parte da chuva de sementes. Assim, estudos sobre as densidades da chuva de sementes podem fornecer informações importantes para a conservação e manejo dos fragmentos florestais. O objetivo desse trabalho foi avaliar a chuva de sementes em um fragmento de floresta atlântica semidecídua, no sul de Minas Gerais, com o intuito de: identificar as espécies e frequências das sementes que foram dispersas; e estabelecer relações da ocorrência dessas sementes com o efeito de borda no fragmento, em diferentes épocas. Foi utilizado um modelo aninhado de análise de variância. Verificaram-se diferenças nas frequências entre as épocas e entre borda e interior, para algumas espécies. As sementes mais frequentes ao longo das coletas foram de Styrax sp., Ficus sp., Copaifera langsdorffii, Siparuna guianensis, Byrsonima crassifolia, Duguetia lanceolata.

Palavras-chave: Fragmento florestal, Mata Atlântica e chuva de sementes.

\section{SEED RAIN IN A SEMIDECIDUOUS ATLANTIC FOREST FRAGMENT}

\begin{abstract}
Survival and dynamics of forests depend largely on the seed rain. Thus, studies on the densities of seed rain can provide important information for the conservation and management of forest fragments. The aim of this study was to evaluate the seed rain in a fragment of semideciduous Atlantic forest in southern Minas Gerais, Brazil, in order to: identify the species and frequency of seeds that were dispersed; and relations between the occurrence of seeds and the edge effect of the fragment, at different times. We used a nested model analysis of variance. We verified differences in frequency among times and between interior and edge, for some species. The most frequent species over the collections were Styrax sp., Ficus sp., Copaifera langsdorffii, Siparuna guianensis, Byrsonima crassifolia, Duguetia lanceolata.
\end{abstract}

Keywords: Forest fragment, Atlantic forest, seed rain. 


\section{INTRODUÇÃO}

A Mata Atlântica é um dos ecossistemas mais ameaçados do mundo (RANTA et al., 1998) e atualmente, os 7,5\% que permanecem da cobertura original dessa floresta no sudeste do Brasil (MYERS et al., 2000) são representados em grande extensão por florestas secundárias (BROWN; BROWN, 1992). Esse bioma consiste em sua maior parte de pequenos fragmentos florestais isolados onde a intensa intervenção antrópica continua a colocar em risco a sua biodiversidade (OLMOS; GALETTI, 2004, apud HIROTA, 2005).

As formações florestais do Brasil, em geral, sofrem considerável degradação, devido ao avanço da fronteira agropecuária ou ainda com a construção de usinas hidrelétricas (NASCIMENTO; SADDI, 1992; apud ALMEIDA et al., 2004). A exploração florestal no sul de Minas Gerais sempre foi intensa. $\mathrm{Na}$ área mais próxima ao reservatório de Furnas, a cobertura nativa não ultrapassa a $6 \%$ e é composta por fragmentos, na sua maioria, inferiores a 30 ha (CAMPOS; LANDGRAF, 2001).

Essas ilhas de vegetação ficam expostas à insolação e à modificação do regime de ventos, o que provoca enorme impacto sobre os organismos que vivem nesses ambientes (LAURANCE; DELAMÔNICA, 1998). Conhecer os efeitos sobre o processo de fragmentação florestal permite planejar a ocupação humana na região e a distribuição dos remanescentes de floresta, de modo a manter uma boa parte da diversidade de espécies (DELAMÔNICA; LAURENCE; LAURENCE, 2001)

Segundo Barbosa e Barbosa (1998) é necessário que se busquem alternativas de manejo para os remanescentes de florestas tropicais, circunstanciadas em objetos concretos de preservação da flora e fauna.

Em florestas tropicais, pode-se observar a existência de uma íntima relação entre dispersão de semente e o processo de regeneração natural de fragmentos florestais. (GARWOOD, 1989). De modo geral, pode-se dizer que a dispersão influencia nos padrões de deposição das sementes, dessa forma, a sobrevivência e a dinâmica das florestas dependem em grande parte do aporte de sementes determinado pela chuva de sementes (HARPER, 1977).

É por meio da chuva de sementes que o banco de sementes e de plântulas está sempre se renovando, permitindo o fechamento de uma clareira, bem como a regeneração natural de uma área degradada (GROMBONE-GUARATINI; RODRIGUES, 2002 apud CAMPOS; SOUZA, 2003).

As observações sobre a chuva de sementes podem fornecer informações úteis sobre a abundância, distribuição espacial, densidade e riqueza de espécies (GROMBONE-GUARATINI; RODRIGUES, 
2002). Assim, os estudos das densidades da chuva de sementes são fontes de informações importantes para a conservação e manejo dos fragmentos.

O objetivo desse trabalho foi avaliar a chuva de sementes em um fragmento florestal no sul de Minas Gerais. Mais especificamente: (i) identificar as sementes que foram dispersas; (ii) determinar a frequência de dispersão em três diferentes épocas do ano (Fevereiro, Abril e Junho); e (iii) estabelecer relações da ocorrência dessas sementes com o efeito de borda no fragmento, nas diferentes épocas.

\section{MATERIAIS E MÉTODOS}

O Fragmento Florestal analisado localiza-se próximo da cidade de Alfenas, sul de Minas Geral, ao lado da rodovia BR 491 (entre Alfenas e Areado). Com uma altitude média de $839 \mathrm{~m}$, o fragmento se localiza na latitude $21^{\circ} 25^{\prime} 27.5^{\prime \prime} \mathrm{S}$ e longitude $46^{\circ} 07^{\prime}$ 29.91" O. A área de estudo é um fragmento de Floresta Atlântica Semidecídua, com clima moderado úmido, temperatura média anual em torno de $23^{\circ} \mathrm{C}$ e precipitação média anual de $1.513 \mathrm{~mm}$ (COSTA, 1998).

A paisagem encontra-se em estado de grande fragmentação, com aproximadamente $4 \%$ de cobertura vegetal nativa, sendo a matriz composta por plantações de café, de cana e pasto (HIROTA, 2009). Em termos de vegetação, o fragmento encontra-se na área de transição entre os biomas Mata Atlântica e Cerrado (RABELLO; RAMOS; HASUI, 2010 apud OLIVEIRA; FERREIRA 2008), porém com a vegetação remanescente encontrando-se extensamente alterada pelo homem.

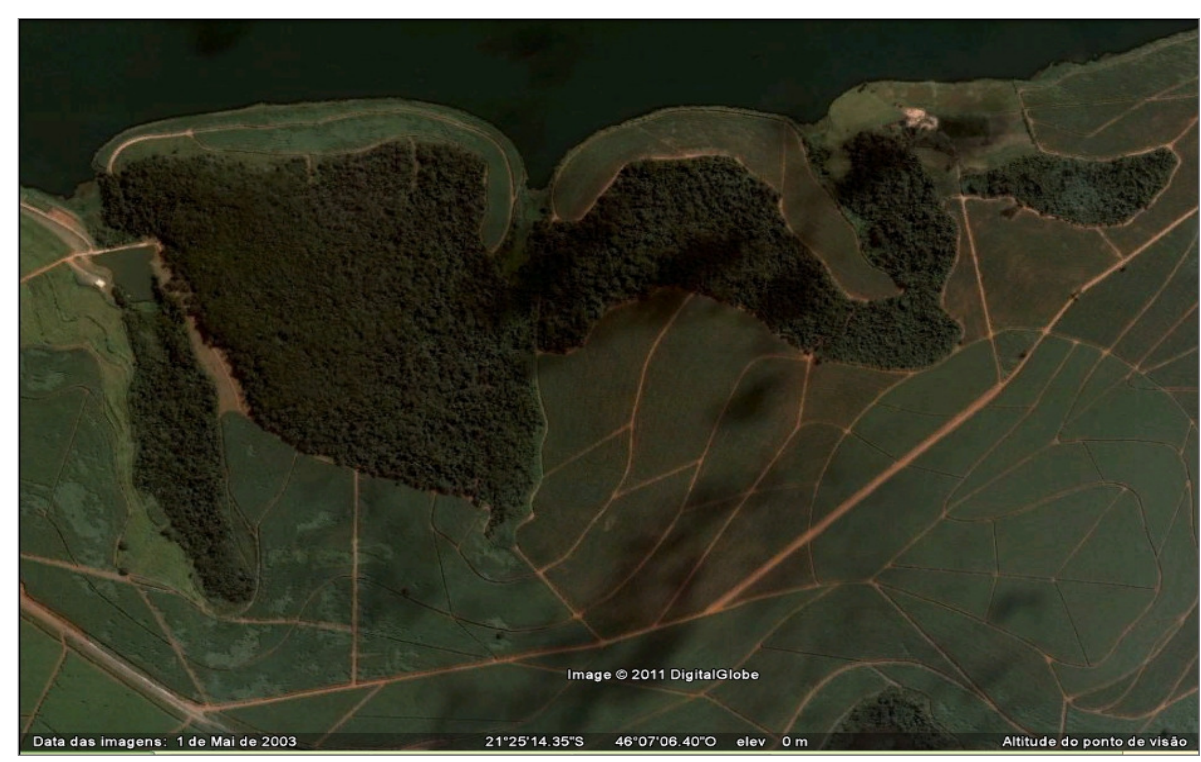

Figura 1. Foto de satélite do fragmento florestal do Porto. Fonte: Google Earth (2011). 
A chuva de sementes foi avaliada por coletores de sementes de $0,25 \mathrm{~m}^{2}$ cada $(50 \times 50$ $\mathrm{cm})$, com altura de $10 \mathrm{~cm}$, que foram distribuídos aleatoriamente dentro de dez parcelas, sendo cinco na borda e cinco no interior. Cada uma das parcelas de $200 \mathrm{~m}^{2}$ (10x20 m) foi subdividida em cinqüenta sub parcelas de $4 \mathrm{~m}^{2}(2 \times 2 \mathrm{~m})$ cada. Dentro de cada parcela foram sorteadas dez sub parcelas onde foram instalados dez coletores de semente. A coleta do material depositado foi realizada a cada dois meses, durante o período de fevereiro a junho.

O material coletado foi levado ao Laboratório de Botânica da Unifal-MG, onde foi triado. Na primeira parte da triagem, o material mais grosso, como galhos e folhas, foram removidos. O material restante foi inspecionado e todos os propágulos acima de $1 \mathrm{~mm}$ de comprimento foram contados e classificados. Foram considerados somente os propágulos maduros e visualmente saudáveis, que não se quebravam sob pressão. A identificação foi realizada de acordo com Lorenzi, Árvores Brasileiras: manual de identificação e cultivo de plantas arbóreas nativas do Brasil.

Após a identificação e contagem dos propágulos, os bancos de dados foram construídos e as tabelas de frequência percentual das espécies mais incidentes na borda e no interior (número de propágulo maior que 100 sementes/parcela) e os setogramas para as espécies menos frequentes foram realizados

A fim de comparar as frequências (apenas feito para as espécies mais frequentes) de dispersão nas diferentes épocas, locais (borda e interior) e investigar a interação entre eles, foi considerado o seguinte modelo aninhado de análise de variância:

$$
y_{i j k l}=\mu+\alpha_{i}+\beta_{j}+\alpha_{i} \beta_{j}+\tau_{k}\left(\beta_{j}\right)+e_{i j k l}
$$

em que $y_{i j k l}$ é o número de sementes observado no $l$-ésimo coletor da $k$-ésima parcela do local $j$, na época $i ; \mu$ é uma constante comum a todas as observações; $\alpha_{i}$ é o efeito da $i$-ésima época; $\beta_{j}$ é o efeito do $j$-ésimo local; $\alpha_{i} \beta_{j}$ é a interação da época $i$ com o local $j ; \tau_{k}\left(\beta_{j}\right)$ é o efeito da $k$ ésima parcela dentro do local $j$; e $e_{i j k l}$ é o erro associado a $y_{i j k l}$. Além disso, considerou-se que $e_{i j k l} \sim N\left(0, \sigma_{e}^{2}\right)$, sendo esses erros independentes e identicamente distribuídos, com $i=1,2$ e 3 ; $j=1,2 ; k=1,2, \ldots, 5$; e $l=1,2, \ldots, 10$. Para as fontes de variação significativas na análise de variância foi aplicado o teste de Tukey a 5\% de significância.

\section{RESULTADOS E DISCUSSÃO}

De acordo com as análises dos dados experimentais pode-se afirmar que as sementes mais frequentes ao longo das três coletas foram das espécies Styrax sp. (9579), Ficus sp. 
(1599), Copaifera langsdorffii (669), Siparuna guianensis (588), Byrsonima crassifolia (460), Duguetia lanceolata (425), além de uma espécie não foi identificada (NI1) (1838). Esses dados também são corroborados por Pimenta (2011), que observou em seu trabalho na região, a maior incidência das espécies Copaifera langsdorffii e Siparuna guianensis.

$\mathrm{Na}$ coleta de Fevereiro apareceram seis das sete espécies mais frequentes (Copaifera langsdorffii, Siparuna guianensis, Duguetia lanceolata, Styrax sp., Ficus sp., NII) sendo que Duguetia lanceolata e Ficus sp. aparecem com maior frequência no interior do fragmento do que na borda, ao contrário das outras quatro (Tabela 1). A Byrsonima crassifolia que é uma das espécies mais frequentes nesta coleta foi agrupada na classe das espécies menos frequentes nas outras coletas.

Tabela 1. Espécies mais frequentes na coleta de Fevereiro.

\begin{tabular}{cccccccc}
\hline FEV & \multicolumn{7}{c}{ Frequência Fp (\%) } \\
\hline Classes & Styrax sp. & NI1 & Ficus sp. & S. guianensis & D. lanceolata & C. langsdorffii & Outras \\
\hline Interior & $14,17 \%$ & $7,43 \%$ & $100 \%$ & $12,22 \%$ & $67,44 \%$ & $13,27 \%$ & $59,05 \%$ \\
\hline Borda & $85,83 \%$ & $92,57 \%$ & $0 \%$ & $87,78 \%$ & $32,56 \%$ & $86,73 \%$ & $40,95 \%$ \\
\hline $\mathrm{N}$ & 8702 & 686 & 620 & 303 & 301 & 211 & 232 \\
\hline
\end{tabular}

Na coleta de Abril apareceram todas as espécies mais frequentes, destas, apenas Byrsonima crassifolia, Ficus sp. e Styrax sp. aparecem com mais frequência no interior do que na borda (Tabela 2).

Tabela 2. Espécies mais frequentes na coleta de Abril.

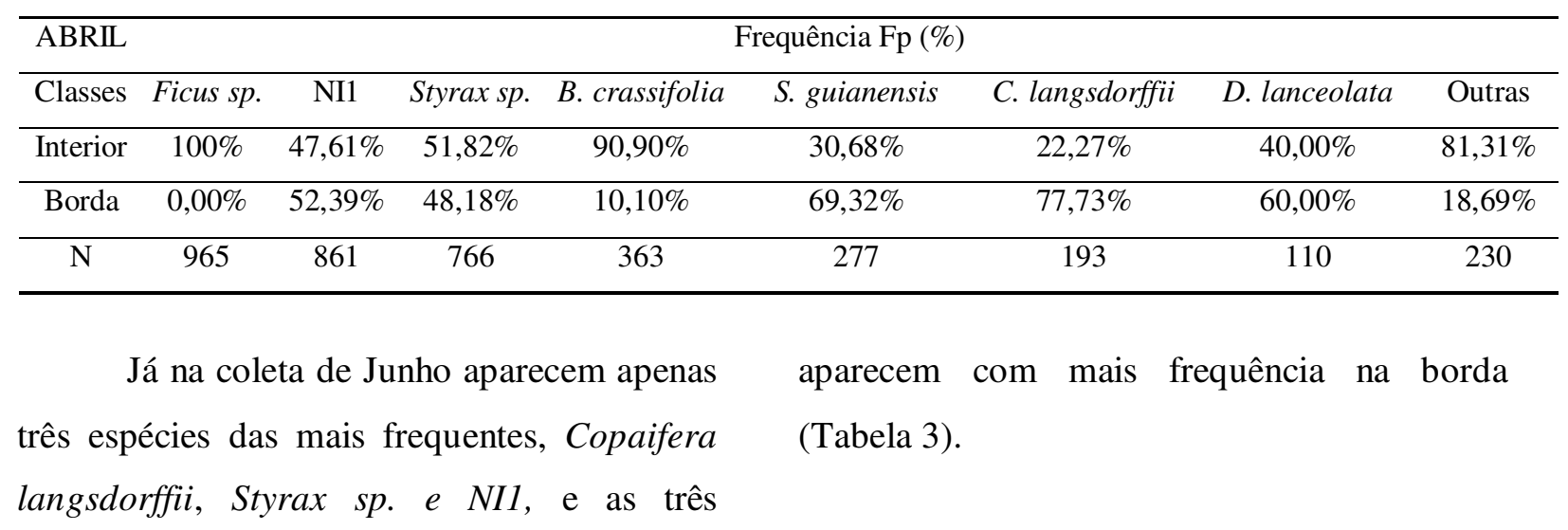


Tabela 3. Espécies mais frequentes na coleta de Junho.

\begin{tabular}{ccccc}
\hline JUNHO & \multicolumn{5}{c}{ Frequência Fp (\%) } \\
\hline Classes & NI1 & Copaifera langsdorffii & Styrax sp. & Outras \\
\hline Interior & $31,95 \%$ & $22,26 \%$ & $11,71 \%$ & $84,15 \%$ \\
\hline Borda & $68,05 \%$ & $77,74 \%$ & $88,29 \%$ & $15,85 \%$ \\
\hline $\mathrm{N}$ & 291 & 265 & 111 & 246 \\
\hline
\end{tabular}

De acordo com o teste de Tukey a 5\% de significância, a espécie Copaifera langsdorffii ocorre mais na borda do que no interior, independente da época testada.

A Copaifera langsdorffii, assim como outras espécies que florescem de Janeiro a Abril e frutificam em Maio-Junho, necessitam de um tempo maior para maturação dos frutos durante a seca, iniciando sua dispersão quando começa o período chuvoso, onde encontrarão condições favoráveis à sua germinação (DIAS; OLIVEIRA-FILHO, 1996). .

Apesar de ter sido possível a observação das sementes de Copaifera langsdorffii em todas as épocas, e ainda ter classificado esta espécie como uma das mais frequentes, essa espécie apresenta um lapso na sua floração e frutificação, esse lapso sugere certa tendência desta espécie em apresentar um padrão trienal de reprodução. (DIAS; OLIVEEIRA-FILHO, 1996)

As sementes de Styrax sp. analisadas, teve maior incidência em Fevereiro, 8702 propágulos, sendo que a maioria apareceram na borda; no interior, a dispersão foi igual em todas as épocas testadas. Analisando as épocas de coleta pode-se dizer que em Fevereiro a dispersão é maior na borda e em Abril e Junho a dispersão é a mesma nas duas regiões.

O gênero Styrax possui cerca de 120 espécies distribuídas nas regiões tropicais e subtropicais, como no Sudeste da Ásia, Oeste da Malásia e Américas (HUTCHINSON, 1973; NAKAJIMA; $\quad$ MONTEIRO, 1986; DICKISON, 1993). No Brasil, são encontradas cerca de 25 espécies deste gênero (NAKAJIMA; MONTEIRO, 1986) , com distribuição geográfica restrita aos estados de Minas Gerais, São Paulo, Mato Grosso do Sul e Paraná (LORENZI, 1992). A maioria das espécies nativas de Styrax ocorre em áreas de cerrado, sendo $S$. ferrugineus e $S$. camporum duas das espécies mais comuns neste ecossistema. Nas matas ciliares, Styrax pohlii passa a ser mais comum (SOUZA; LORENZI, 2005).

A espécie Siparuna guianensis, no interior, apresentou a mesma dispersão em todas as épocas assim, como a Styrax sp; enquanto na borda, as maiores dispersões aconteceram em Fevereiro e Abril. Nos meses de fevereiro e abril a dispersão foi maior na borda, e em Junho, a dispersão foi igual no interior e na borda. 
$O$ gênero Siparuna ocorre na maioria dos tipos de vegetação neotropical em elevações entre o nível do mar e 3800 metros (SOUZA; LORENZI, 2005), foi classificado como de clímax exigente de luz por Davide et al. (1995) e Ressel et al. (2004); como de clímax tolerante à sombra por Nunes et al. (2003) e Pinto et al. (2005), e como secundário por Souza et al. (2006).

A grande quantidade de sementes de Siparuna guianensis tanto na borda quanto no interior pode ser resultado de sua plasticidade, pois ela ocorre tanto em ambientes sombreado quanto em ambientes com maior luminosidade (PEZZOPANE, 2002).

As análises de variância da espécie Byrsonima crassifolia indica que na borda a dispersão ocorre igualmente em todas as épocas e no interior a dispersão foi maior em Abril. Além disso, nos meses de Fevereiro e Junho a dispersão foi igual no interior e borda.

Segundo Pereira e Freitas (2002) a frutificação dessa espécie tem seu início no final de Dezembro, prolongando-se até o final de Abril, o que corrobora o resultado encontrado neste trabalho, presença de sementes de Byrsonima crassifólia em Fevereiro e Abril.
A dispersão de sementes da espécie Duguetia lanceolata, e da espécie denominada NI1, foi à mesma em todas as épocas testadas, tanto na borda quanto no interior.

As espécies classificadas como menos frequentes, foram agrupada em uma classe denominada "Outras", como pode ser observado nas tabelas de distribuições de frequência (tabelas 1, 2 e 3). Foram identificadas 18 espécies diferentes ao longo das coletas realizadas. Essas espécies são: Xylopia brasiliensis, Sebastiania brasiliensis, Ilex paraguariensis, Maprounea guianensis, Cissampelos ovalifolia, Cryptocarya aschersoniana, Cecropia pachystachya, Amaioua guianensis, Aegiphila sellowiana, Desmodium adscendens, Platypodium elegans, Casearia arborea, Platycyamus regnelli, Croton sp., Geonoma sp., Trichila sp., Protium sp. e Não identificada 2 (NI2).

No interior, em Fevereiro, as espécies Byrsonima crassifolia, Cissampelos ovalifolia, Protium sp., Maprounea guianensis, Cecropia pachystachya foram as mais frequentes, assim como Sebastiania brasiliensis e Xylopia brasiliensis que nesta época apareceram somente nesta região. (Figura 2). 


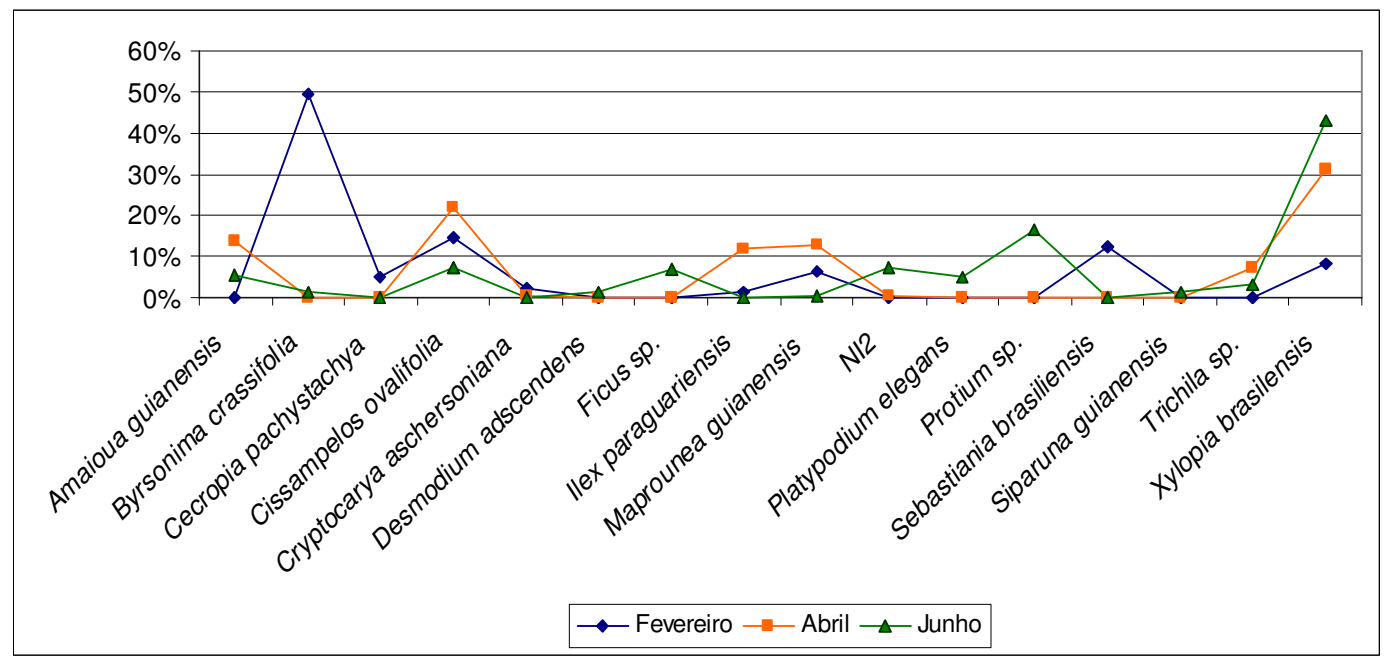

Figura 2. Espécies menos frequentes no interior do fragmento, nas três coletas.

A Sebastiania brasiliensis é por descrita por Smith et al. (1988) como espécie frequente nos sub-bosques dos pinhais, adaptada a solos úmidos e situadas em pequenas depressões, sendo seletiva higrófila e adaptada à luz difusa. Segundo Narvaes, Longhi e Brena (2008) a Sebastiania brasiliensis esta adaptada à posição topográfica intermediária (encosta média) onde existem boas condições de umidade, acarretada em especial pela baixa declividade.

\section{A Xylopia brasiliensis costuma} apresentar a floração no início das primeiras chuvas, e apresentar um longo período de frutificação iniciando-se no final da estação chuvosa e estendendo-se pela estação seca (DIAS; OLIVEIRA-FILHO, 1997).

Em Abril, o grupo "Outras", no interior, apresentou maior frequência de sementes das espécies Amaioua guianenses, Cissampelos ovalifolia, Ilex paraguariensis,
Maprounea guianensis, Trichila sp e Xylopia brasiliensis. A dispersão de sementes da espécie Cissampelos ovalifolia nesta época ocorreu exclusivamente nesta região (Figura 2).

Segundo Dias e Oliveira-Filho (1997) a floração de Amaioua guianensis tende a se comportar de maneira semelhante à de Xylopia brasiliensis no que se refere à estação, ou seja, em ambas a floração parece ser iniciada pelas primeiras chuvas, provavelmente é por isso que ambas as espécies apresentou dispersão de sementes na mesma época.

A tendência de florir no início da estação chuvosa parece ser comum em regiões tropicais com clima sazonal, como observado na vegetação tropical semiárida da Venezuela (LAMPE, 1992), na floresta decídua do México (BULLOCK; $\quad$ SOLISMAGALLANES, 1989); na floresta pluvial tropical das Filipinas (HEIDMAN, 1989), na 
floresta ombrófila sub-montana de Santa Teresa-ES (JACKSON, 1978), na floresta pluvial de Sumatra (VAN SCHAIK, 1986), e na floresta de altitude em Jundiaí-SP (MORELLATTO, 1989).

Em Junho, as mais frequentes foram Amaioua guianensis, Cissampelos ovalifolia, Protium sp. e Xylopia brasiliensis. A espécie Xylopia brasiliensis foi a que apareceu em maior abundância, essa espécie foi mais comum no interior do fragmento do que na borda, assim como ocorreu nas coletas de Fevereiro e Abril (Figura 2).

No interior, ao longo de todas as coletas, as maiores quantidades de propágulos foram das espécies Byrsonima crassifolia e Xylopia brasiliensis. Porém a primeira espécie apresentou grande quantidade de propágulos em uma única coleta e um número reduzido nas outras, já a segunda espécie dispersou um número crescente de sementes no decorrer das três coletas (Figura 2).

Analisando as coletas feitas na borda do fragmento pode-se observar que em Fevereiro as sementes mais frequentes foram das espécies Byrsonima crassifolia, Ilex paraguariensis, Cecropia pachystachya e Croton sp. As sementes de Croton sp., em todas as coletas, apareceu exclusivamente na borda, e em Fevereiro representou $45 \%$ das sementes que apareceram nesta região (Figura $3)$.

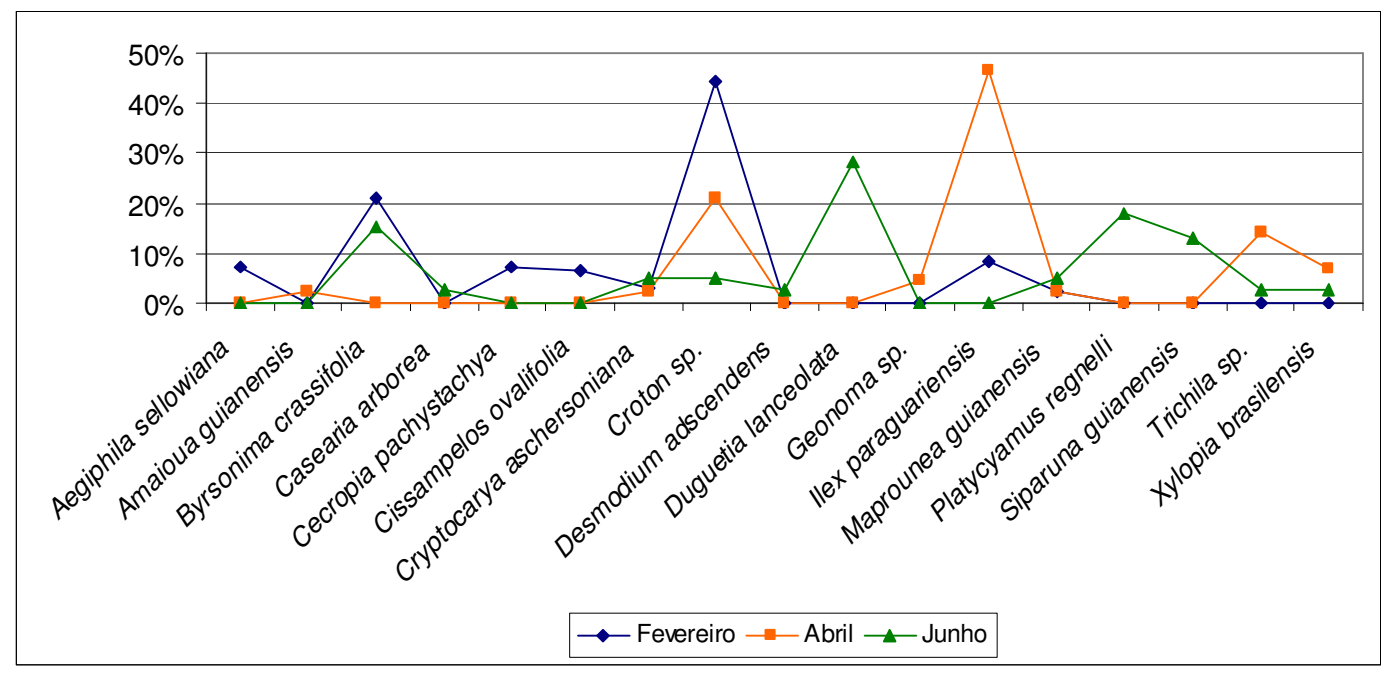

Figura 3. Espécies menos frequentes na borda do fragmento, nas três coletas. 
A identificação de dispersão de sementes de Ilex paraguariensis em Fevereiro e Abril também foi evidenciada por Liebsch e Mikich (2009) que relata ter observado a dispersão de sementes maturas na época de Janeiro a Junho.

O estudo sobre a dispersão de sementes de Cecropia pachystachya é de grande importância, pois essa espécie é ótima para ser usada em bordas de fragmentos em processo de regeneração natural. Ela apresenta crescimento vigoroso e sombra frondosa, capaz de competir com espécies em desequilíbrio e espécies invasoras (ATTANASIO et al., 2006), assim como as espécies Croton sp., Platypodium elegans, Ficus sp.

$\mathrm{Na}$ coleta de Abril, as sementes mais abundantes na borda continuaram sendo das espécies Ilex paraguariensis e Croton sp., porém o número de sementes de Croton $s p$. reduziu quase pela metade, e o número de sementes de Ilex paraguariensis aumentou de $8 \%$ para $46 \%$, de Fevereiro para Abril (Figura $3)$.

Em Junho, as sementes de Duguetia lanceolata, Byrsonima crassifolia, Platycyamus regnelli, Siparuna guianensis, Maprounea guianensis foram mais abundantes na borda (Figura 3), ao contrário do que ocorreu em Fevereiro e Abril onde as sementes Byrsonima crassifolia e Maprounea guianensis foram mais abundantes no interior.
A presença de Croton sp. também foi observada nesta região, porém com um número de propágulos ainda mais reduzido.

$\mathrm{Na}$ coleta de Junho a diversidade de espécies foi maior do que nas outras coletas analisadas, além disso, a composição do grupo "Outras" diverge quando se compara interior e borda. As sementes de Cissampelos ovalifolia, Amaioua guianensis, Platypodium elegans, Ficus sp. e Protium sp. aparecem apenas no interior, e as sementes de Platycyamus regnelli, Casearia arbórea, Cryptocarya aschersoniana e Croton sp. na borda.

Segundo Attanasio et al. (2006) as espécies Duguetia lanceolata, Ilex paraguariensis, Protium sp., Copaifera langsdorffii, Casearia arborea, Byrsonima crassifolia e Ficus sp. são de suma importância, pois são espécies atrativas para a fauna, visando à manutenção e a introdução de polinizadores e dispersores . Esse fato contribui para aumentar a diversidade vegetal e animal de um fragmento florestal.

\section{CONCLUSÕES}

Conclui-se, portanto, que no fragmento estudado a maioria das espécies que apresentaram maior incidência e dispersaram mais sementes na borda do que no interior, com exceção das sementes da espécie Duguetia lanceolata e NIl, que apresentaram dispersão igual em todas as 
épocas, e da espécie Ficus sp. que apareceu

predominantemente no interior.

O grupo das espécies menos frequentes, apesar de não apresentar grande quantidade de sementes, apresentou grande diversidade de espécies. Além disso, as sementes dispersadas por esse grupo foram mais comuns no interior do que na borda.

\section{AGRADECIMENTOS}

Agradecemos à colaboração da Prof. Dr. Julia Caram Sfair - UNICAMP-SP na identificação das sementes, à Unifal-MG que nos cedeu o transporte aos fragmentos, ao proprietário do fragmento florestal Sr. Miguel A. C. Barbosa pela permissão concedida e à CAPES pela bolsa de mestrado concedida a F. F. Silva.

\section{REFERÊNCIAS BIBLIOGRÁFICAS} ALMEIDA-CORTEZ， J. S. Dispersão e banco de sementes. In: FERREIRA, A. G.; BORGHETTI, F. (Orgs.). Germinação: do básico ao aplicado. Porto Alegre: Artmed, 2004. p. 225-235.

ATTANASIO, C. M. et al. Adequação ambiental de propriedades rurais, recuperação de áreas degradadas e restauração de matas ciliares. Piracicaba: ESALQ, Departamento de Ciências Biológicas, Laboratório de Ecologia e Restauração Florestal, 2006. 63 p.
BARBOSA, L. M.; BARBOSA, J. M. Informações técnico-científicas sobre Recuperação de Áreas Degradadas. Revista Ecossistema, Espírito Santo do Pinhal, v.23, p. 19-25, 1998.

BROWN, JR. K. S.; BROWN, G. G. Habitat alteration and species loss in Brazilian forests. In: TC Whitmore \& JA Sayer (eds.). Tropical deforestation and species extinction. London: Chapman \& Hall, 1992.

BULLOCK, S. H.; SOLIS-MAGALLANES, J. A. Phenology of canopy trees of a tropical deciduous forest in México. Biotropica, Kansas, 1989.

CAMPOS, J. C.; LANDGRAF, P. R. C. Análise da regeneração natural de espécies florestais em matas ciliares de acordo com a distância da margem do lago. Ciência Florestal, v. 11, n. 2, p.143-151, 2001.

CAMPOS, J. B.; SOUZA, M. C. Potential for Natural Forest Regeneration from Seed Bank in an Upper Paraná River Floodplain, Brazil. Brazilian Archives of Biology and Tecnology, v. 46, n. 4, p. 625-639. 2003.

COSTA, C. M. R. Biodiversidade em Minas Gerais: um atlas para a sua conservação. 
Fundação Biodiversitas, Belo Horizonte. 1998.

DAVIDE, A.C. et al. Propagação de espécies florestais. Belo Horizonte: CEMIG/UFLA/FAEPE, 1995. 41 p.

DELAMÔNICA， P; LAURENCE，W. F.; LAURENCE, S. G. A fragmentação da paisagem. In: Florestas do Rio Negro. Companhia das letras, 2001, cap.9:, p. 283.

DIAS, H. C. T.; OLIVEIRA-FILHO, A. T. de. Fenologia de quatro espécies arbóreas de uma floresta estacional semidecídua montana em Lavras, MG. Cerne, Lavras, DCF/UFLA, v. 2, n. 1, p. 66-88, 1996.

DIAS, H. C. T.; OLIVEIRA-FILHO, A. T. de. Variação temporal e espacial da serapilheira em uma área de floresta estacional semidecídua montana em Lavras, MG. Revista Árvore, Viçosa, SIF/UFV, v. 21, n. 1, 1997.

DICKISON, W. C. Floral anatomy of the Styracaceae, including observations on intraovarian trichomes. Botanical Journal of the Linnean Society, v. 112, p. 223-255, 1993.

GARWOOD, N. C. Tropical soil seed banks: a review. p. 149-209. In: LECK, M. A.; PARKER, V. T.; SIMPSON R. L. (eds.).
Ecology of soil seed banks. San Diego: Academic Press, 1989.

GOOGLE EARTH. Disponível em: $<$ http://www.google.com/intl/pt-PT/earth/>. Acesso em: 24 Nov 2011.

GROMBONE-GUARANTINI, M. T.; RODRIGUES, R. R. Seed bank and seed rain in a seasonal semi-deciduous forest in SouthEastern Brazil. Journal of Tropical Ecology, Winchelsea, v. 18, p. 759-774, 2002.

HARPER, J. L. Population biology of plants. London: Academic Press, 892 p., 1977.

HEIDEMAN, P. D. Temporal and spatial variation in the phenology of flowering and fruiting in a tropical rainforest. Journal of Ecology, New York, v.17, p.1059-1079, 1989.

HIROTA, M. M. Monitoramento da cobertura da Mata Atlântica brasileira. In: GALINDOLEAL, C.; CÂMARA, I. G. (eds.), Mata Atlântica: biodiversidade, ameaças e perspectivas. Fundação SOS Mata Atlântica. BH. Conservação Internacional, Cap. 6. p.6065. 2005.

HIROTA, M. M. Atlas dos remanescentes florestais da Mata Atlântica: Período 20082010. Fundação SOS Mata Atlântica e 
Instituto Nacional de Pesquisas Espaciais. São

Paulo. 2009. 60 p.

HUTCHINSON, J. The families of flowering plants. $3^{\text {rd }}$ ed. Clarendon Press, Oxford. 1973.

JACKSON, J. F. Seasonality of flowering and leaf-fall in a Brasilian subtropical lower montane moist forest. Biotropica, Kansas, v.10, n.1, mar. 1978.

LAMPE, M. G. de et al. Seasonal flowering and fruiting patterns in tropical semi-arid vegetation of Northeastern Venezuela. Biotropica, Kansas, v. 24, n. 1, p. 64-76, 1992.

LAURANCE，W. F.; DELAMÔNICA， P. Ilhas de sobrevivência: Ecologia e dinâmica de fragmentos florestais na Amazônia. Revista Ciência Hoje, v. 24, n. 142, p. 26-31. 1998.

LIEBSCH, D. ; MIKICH, S. B.: Fenologia reprodutiva da Floresta Ombrófila Mista do Paraná, Brasil. Revista Brasil. Bot., v. 32, n. 2, p. 375-391. 2009.

LORENZI, H. Árvores brasileiras: manual de cultivo e identificação de plantas arbóreas nativas do Brasil. Nova Odessa: Editora Plantarum. 1992.
MYERS, N. et al. Biodiversity hotspots for conservation priorities. Nature, v. 403, p. 853-858, 2000.

MORELLATO, L. P. C. et al. Estudo comparativo da fenologia de espécies arbóreas de floresta de altitude e floresta mesófila semidecídua na Serra do Japi, Jundiaí, São Paulo. Revista Brasileira de Botânica, São Paulo, v. 12, n. 1/2, p. 85-98, dez. 1989.

NAKAJIMA, J. N. e MONTEIRO, R. Estudos fitogeográficos com espécies de Styrax L. (Styracaceae) dos cerrados brasileiros. Eugeniana, v. 12, p. 3-10. 1986.

NASCIMENTO, M.T.; SADDI, N. Structure and composition of cerrado in Cuiabá-MT, Brazil. Revista Brasileira de Botânica, São Paulo, v. 15, n. 1, p. 47-55, 1992.

NARVAES, I. S., LONGHI, S. J. , BRENA, D. A. Florística e classificação da regeneração natural em Floresta Ombrófila Mista na Floresta Estacional de São Francisco de Paula. Ciência Florestal, Santa Maria, v. 18, n. 2, p. 233-245, abr.-jun, 2008.

NUNES, Y. R. F. et al. Variações da fisionomia, diversidade e composição de guildas da comunidade arbórea em um 
fragmento de floresta semidecidual em Lavras, MG. Acta Botanica Brasílica, v. 17, n. 2, p. 213-29, 2003.

OLIVEIRA FILHO, A. T.; SCOLFORO, J. R. S.; MELLO, J. M. de. Composição florística e estrutura comunitária de um remanescente de floresta semidecídua montana em Lavras, MG. Revista Brasileira de Botânica, São Paulo, v. 17, n. 2, p. 167-182, dez. 1994.

OLMOS, F.; GALETTI, M. A conservação e o futuro da Juréia: isolamento ecológico e impacto humano. In MARQUES, O. A. V.; DULEBA, W. Estação Ecológica JuréiaItatins: Ambiente físico, flora e fauna. Editora Holos, Ribeirão Preto, p. 360-377, 2004

OLIVEIRA, R. L. S.; FERREIRA, M. F. Unidades de paisagem da região de Alfenas, sul de Minas Gerais, a partir de dados do radar SRTM e imagem orbital ETM + LANDSAT 7 utilizando a abordagem geossistêmica. In I Semana de Geotecnologias. (CENTRO DE ANÁLISE E PLANEJAMENTO AMBIENTAL CEAPLA, ed.) Rio Claro, 2008.

PEREIRA, J. O. P.; FREITAS, B. M. Estudo da biologia floral e requerimento de polinização do muricizeiro (Byrsonima crassifolia, L.). Revista Ciência
Agronômica, Fortaleza-CE, v. 33, n. 2, p. 5560, 2002.

PEZZOPANE, J. E. M.; REIS, G. G.; REIS, M. G. F.; HIGUSHI, P.; POLLI, H. Q. Aspectos ecofisiológicos de algumas espécies arbóreas em uma floresta estacional semidecidual secundária. Revista Brasileira de Agrometereologia, v. 10, n. 2, p. 273-281, 2002.

PINTO, L. V. A. et al. Estudo da vegetação como subsídios para propostas de recuperação das nascentes da bacia hidrográfica do Ribeirão Santa Cruz, Lavras, MG. Revista Árvore, v. 29, n. 5, p. 775-93, 2005.

PIMENTA, E. P. Comparação da comunidade arbórea em fragmentos florestais rodeados por diferentes tipos de matriz. 2011. 45 p. Trabalho de Conclusão de Curso - Faculdade de Ciências Biológicas, Universidade Federal Alfenas, Alfenas-MG, 2011.

RABELLO, A.; RAMOS, F.N.; HASUI, E. Effect of fragment size on Copaifera langsdorffii seeds dispersal. Biota Neotop, v. 10, n. 1, 2010.

http://www.biotaneotropica.org.br/v10n1/en/a bstract?article+bn00810012010. 
RANTA, P. et al. The fragment Atlantic forest of Brazil: size, shape and distribution of forest fragments. Biodiversity Conservation, v. 7, p. 385-403. 1998.

RESSEL, K. et al. Ecologia morfofuncional de plântulas de espécies arbóreas da Estação Ecológica do Panga, Uberlândia, Minas Gerais. Revista Brasileira de Botânica, v. 27, p. 311-323. 2004.

SMITH, L. B.; DOWNS, R. J.; KLEIN, R. M. Euforbiáceas In: REITZ, P. R. (Ed.). Flora ilustrada Catarinense .Itajaí: Herbário Barbosa Rodrigues, 1988. 408 p.

SOUZA, C. D.; FELFILI, J. M. Uso de plantas medicinais na região de Alto Paraíso de Goiás, GO, Brasil. Acta Botanica Brasílica, v. 20, n. 1, p. 135-42, 2006.

SOUZA, V. C.; LORENZI, H. Botânica Sistemática: Guia ilustrado para identificação das famílias de Angiospermas da flora brasileira, segundo A.P.G.II. Nova Odessa: Editora Plantarum, 2005. v. 1. 640 p.

VAN SCHAIK, C. P. Phenological changes in a Sumatran rain forest. Journal of Tropical Ecology, Aberdeen, v. 2, p. 327347, 1986. 